\title{
Influence de l'agitation des échantillons de lait cru sur les résultats de dénombrement de trois groupes microbiens d'intérêt technologique
}

\author{
par \\ Christine PITON* et J. RICHARD
}

\section{Rés u m é}

Quatre-vingt-dix échantillons de lait cru agités de deux manières différentes (simples retournements du flacon ou agitation énergique par turbine) ont été soumis au dénombrement de la flore totale et de trois groupes microbiens d'intérêt technologique : flore thermorésistante totale, bactéries coliformes et flore psychrotrophe résistant à la pénicilline.

En moyenne, l'influence du mode d'agitation sur les résultats de dénombrement est nulle pour la flore thermorésistante. Par contre, elle est importante dans le cas de la flore psychrotrophe résistant à la pénicilline et des bactéries coliformes : les nombres moyens de bactéries dans le lait agité par turbine sont respectivement 4,5 et 2,5 fois plus élevés que dans les échantillons peu agités ; des écarts de 1 à 20 ou même de 1 à 100 ont été observés. Pour la flore totale, l'écart dans les résultats entre les deux méthodes d'agitation est fonction des proportions de micro-organismes thermorésistants et de microorganismes psychrotrophes résistant à la pénicilline dans cette flore.

Mots clés :

Lait cru - Dénombrement microbien - Agitation des échantillons.

Laboratoire de Microbiologie Laitière et de Génie Alimentaire, C.N.R.Z, - 78350 Jouy-en-Josas (France).

* Présente adresse : Institut Technique de l'Elevage Bovin, 149, rue de Bercy 75595 Paris cedex 12. 
INFLUENCE OF TWO SHAKING PROCEDURES ON COUNTS OF THREE GROUPS OF MICRO-ORGANIMS OF TECHNOLOGICAL INTEREST

\section{Su m m a ry}

Ninety samples of raw milk (mixture of 4 milkings) were submitted to two shaking methods: a gentle one consisting in 15 inversions of the bottle containing the cold milk and a vigorous homogenization using a blender (Ultra Turrax, model 18/2) for $30 \mathrm{~s}$ at $20000 \mathrm{rpm}$.

The samples were then submitted to the enumeration of a) the total count on Plate Count Agar after $3 \mathrm{~d}$ of incubation at $30^{\circ} \mathrm{C}, \mathrm{b}$ ) the thermoduric count afer pasteurization of the milk at $63,5^{\circ} \mathrm{C}$ for 30 min and enumeration of the survivals as for total count, c) the coliform bacteria on Violet Red Bile Agar after 18-24 h of incubation at $30^{\circ} \mathrm{C}$ and d) penicillin resistant psychrotrophic (PRP) microorganisms (mostly pseudomonads) on nutrient agar containing 100 $I U / m l$ of benzyl penicillin.

The samples were pour plated after decimal dilution in quater strength Ringer solution or plated without dilution using the Spiral Plater.

The most significant differences between the two shaking procedures was noted for the coliform bacteria and the PRP microorganisms, the counts beeing on average respectively 2.5 and 4.5 times higher with the most efficient method; ratios as high a 100: 1 were not unfrequent. No significant difference was observed for the thermoduric microflora. The lotal count was more or less affected depending on the proportion of thermoduric and PRP micro-organisms in milk.

It is concluded that the milk samples should be vigorously shaken when PRP micro-organisms are enumerated as the true number of pseudomondas determine the keeping quality of the raw milk stored at low temperature.

Key words:

Raw milk - Microbial count method - Sample Shaking.

\section{INTRODUCTION}

L'influence de l'agitation du lait cru sur les résultats de dénombrement de sa flore totale est désormais connue (Wanser et Hartman, 1962 ; Huhtanen et al., 1970 ; Te Whaiti et Fryer, 1977 ; Richard, 1980 ; Richard et al., 1981). Actuellement, c'est probablement l'agitation par turbine qui assure la meilleure dispersion des agrégats microbiens; à l'opposé, une agitation faible consistant, par exemple, en de simples retournements du flacon jusqu'à l'homogénéisation apparente de la 
matière grasse, conduit à une très nette sous-estimation du nombre total de micro-organismes présents dans le lait (Richard et al., 1981).

Il semble cependant que l'influence de l'agitation varie en fonction de la nature de la flore. Ainsi, au cours d'une étude comparative de la méthode classique de numération par dilutions et de la méthode Thompson, Tatini et al. (1967) ont observé que l'écart entre les deux méthodes dépendait de l'origine des échantillons (lait de bidons ou lait de tank) et du niveau de la flore ; ils ont postulé que la nature de la flore était responsable de cet écart : le lait de bidons (probablement du fait qu'il n'était pas conservé au froid) contiendrait en majorité des microcoques et des streptocoques formant des amas ou des chaînes tandis que la flore des laits de tank serait surtout constituée de bactéries psychrotrophes se présentant généralement sous forme de bâtonnets isolés ou par paires. La méthode par dilution permettait d'obtenir des nombres de colonies supérieurs à l'autre méthode car elle assurait une meilleure dispersion des amas microbiens. Or, d'après Richard (1980), les amas formés par les microcoques et les streptocoques paraissent plus difficiles à dissocier que ceux formés par les bâtonnets. D'autre part, cet auteur a observé une interaction « Echantillon - Mode d'agitation » importante attribuée en partie à la nature de la flore ; cependant, il n'a pas pu mettre en évidence, par les dénombrements sélectifs des streptocoques (milieu à l'acétate de thallium), des microcoques (milieu hypersalé de Chapman) et des bactéries à gram négatif (milieu au cristal violet), lequel de ces groupes bactériens était responsable de l'élévation du nombre de colonies après une agitation vigoureuse des échantillons : le nombre de colonies sur les deux premiers milieux restait très faible ; sur le milieu au cristal violet, il était supérieur au témoin mais l'augmentation était insuffisante pour expliquer celle de la flore totale. Par ailleurs, Te Whaiti et Fryer (1977) ont constaté que les bactéries psychrotrophes formaient, au cours de leur mulitiplication, des agrégats microbiens de taille d'autant plus importante que le lait a été agité plus longtemps à basse température ; ils ont observé que l'on pouvait souvent, de ce fait, faire une sous-estimation très importante du nombre de bactéries dans le lait : nombres observés de 5 à 10 fois inférieurs à la réalité pour des échantillons riches en bactéries psychrotrophes. En outre, ils ont montré que l'on pouvait facilement disperser ces agrégats microbiens en refoulant à trois reprises le lait aspiré dans une seringue.

Il nous a semblé intéressant de connaître aussi l'influence de l'agitation du lait cru sur les résultats de dénombrement d'autres groupes microbiens d'intérêt technologique comme la flore thermorésistante totale, les bactéries coliformes et la flore psychrotrophe résistante à la pénicilline. Cette dernière est composée en majorité de Pseudomonas lesquels déterminent la qualité de conservation du lait cru à basse température (Richard, 1981). 


\section{MATERIEL ET METHODES}

\section{Origine des échantillons}

Quatre-vingt-dix échantillons de lait réfrigéré (mélange habituel de 4 traites) ont été prélevés dans différentes fermes et conservés dans la glace fondante jusqu'au moment de l'analyse, au plus tard $24 \mathrm{~h}$ après le prélèvement.

\section{Modes d'agitation}

Chaque échantillon subissait d'abord une agitation douce couramment utilisée dans des laboratoires interprofessionnels qui consiste en 15 retournements du flacon; une fraction de ce lait était ensuite soumise à un traitement de 30 secondes à l'aide d'un homogénéiseur à turbine tournant à $20000 \mathrm{t} / \mathrm{min}$ (appareil Ultra-Turrax 18/2) (Richard, 1980).

\section{Méthodes d'ensemencement}

Pour les 66 premiers échantillons de l'étude, on a utilisé la méthode classique de dénombrement : dilutions décimales et incorporation de l'inoculum dans la gélose (Anon., 1972). Les dilutions ont été réalisées dans du liquide de Ringer dilué au quart ; les tubes de dilution étaient agités 5 à $10 \mathrm{~s}$ à l'aide d'un vibrateur de type Vortex réglé au maximum. Pour chaque échantillon, on a inoculé deux boîtes de Petri à partir du même tube de dilution. Dans la seconde partie de l'étude, le lait a été ensemencé à la surface de la gélose à l'aide d'un ensemenceur en spirale* à raison d'une seule boîte par échantillon. On admettra que le changement de technique d'ensemencement n'affecte pas les résultats de la comparaison. Il a en effet été largement montré que la méthode d'ensemencement en surface donne des résultats similaires à ceux de la méthode classique (Campbell et Gilchrist, 1973 ; Gilchrist et al., 1973 ; Donnelly et al., 1976 ; Jarvis et al., 1977 ; Peeler et al., 1977). Dans les deux cas, le comptage des colonies a été réalisé manuellement.

\section{Dénombrements effectués}

Les échantillons étaient soumis aux dénombrements suivants :

- flore totale sur milieu Plate Count Agar (Difco) ; incubation $3 \mathrm{j}$. ̀̀ $30^{\circ} \mathrm{C}$;

\footnotetext{
* Spiral System Marketing, Interscience, 3, rue de Milan, Paris.
} 
- flore psychrotrophe résistant à la pénicilline, sur milieu Nutrient Agar (Difco) additionné de $100 \mathrm{UI} / \mathrm{ml}$ de pénicilline ; incubation $10 \mathrm{j}$. à $7^{\circ} \mathrm{C}$;

- bactéries coliformes sur milieu Violet Red Bile Agar (Difco) ; incubation 18 à $24 \mathrm{~h}$ à $30^{\circ} \mathrm{C}$; pour les échantillons ensemencés en surface, nous n'avons pas recouvert le milieu d'une seconde couche de gélose (nous avons vérifié par ailleurs que les colonies acidifiantes étaient effectivement des coliformes) ;

- flore thermorésistante : après chauffage du lait à $63,5^{\circ} \mathrm{C}$ pendant $30 \mathrm{~min}$, dénombrement selon la méthode employée pour la flore totale.

\section{RESULTATS ET DISCUSSION}

Les figures 1 à 4 montrent, pour les 3 groupes microbiens d'intérêt technologique et pour la flore totale, l'influence du mode d'agitation sur les résultats de numérations. Pour la flore psychrotrophe résistant à la pénicilline (fig. 1) et les bactéries coliformes (fig. 2), on observe que tous les points sont situés au-dessus de la droite d'équation $\mathrm{Y}=\mathrm{X}$. Les écarts à cette droite sont plus ou moins importants selon l'origine des échantillons; ils sont en général inférieurs à 1,3 unité de $\log$ (à $\mathrm{P}=0,95$ ) avec une moyenne de 0,65 unité de log pour les Pseudomonas et de 0,40 unité de log pour les bactéries coliformes, soit des rapports moyens « nombres de bactéries obtenues avec agitation violente "sur "nombres avec agitation douce " respectivement de 4,5 et 2,5 ; ils peuvent atteindre presque 2 unités de log (rapport égal à 100 !).

Il en résulte que pour certains échantillons, on fera, avec un mode d'agitation peu énergique, une très nette sous-estimation du nombre de ces micro-organismes. Par ailleurs, l'influence de l'agitation des échantillons ne semble pas varier en fonction de leur niveau de contamination : les droites de régression sont en effet sensiblement parallèle à la première bissectrice (pente proche de 1). La grande dispersion des points autour de la droite de régression pour ces deux groupes microbiens peut sans doute être expliquée par une variation de la taille des amas microbiens selon l'origine de l'échantillon : on peut penser que les amas microbiens présents dans le matériel de traite ont subi une dispersion plus ou moins importante au cours de la traite, en particulier par pompage ; mais par la suite, le lait, dans certains cas, a pu être le siège d'une multiplication microbienne et/ou d'une agglutination par les immunoglobulines (Bottazzi et al., 1972).

Pour la flore thermorésistante (fig. 3), tous les points sont situés de part et d'autre de la première bissectrice, ce qui signifie que même 


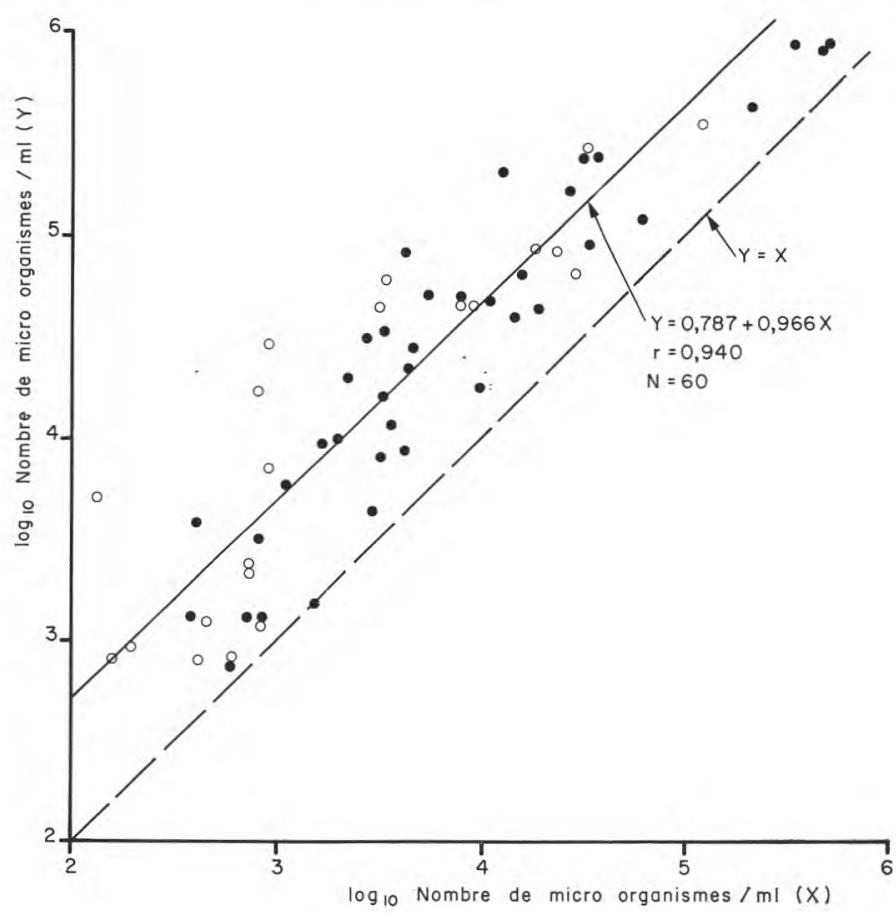

fig. 1

Influence du mode d'agitation des échantillons de lait cru sur les résultats de numération de la flore psychrotrophe résistant à la pénicilline. En abcisse : agitation douce (15 retournements du flacon). En ordonnée : agitation violente (turbine à $20000 \mathrm{t} / \mathrm{min}$ pendant 30 secondes). En noir : ensemencement par dilution ; en blanc : ensemencement en spirale. $\mathrm{r}$ : coefficient de corrélation. $\mathrm{N}$ : nombre d'échantillons.

Influence of the shaking methods on the results of the enumeration of the psychrotroph penicillin resistant bacteria in raw milk. Gentle agitation (abscissa), violente agitation (vertical axis). In black: pour plating, in white: spiral plating.

le mode d'agitation très énergique que nous avons utilisé n'augmente pas significativement les résultats de dénombrement de cette flore.

Comme pour la flore psychrotrophe résistant à la pénicilline et les bactéries coliformes, on observe, pour la flore totale (fig. 4), des écarts systématiques par rapport à la droite d'équation $\mathrm{Y}=\mathrm{X}$. La variabilité de ces écarts peut être attribuée à la nature des bactéries qui composent la flore totale ; en effet, l'influence de l'agitation est d'autant plus importante que les échantillons possèdent une forte proportion de bactéries psychrotrophes et inversement, les points sont plus proches de la bissectrice lorsque la flore thermorésistante du lait est à un niveau élevé. Il en résulte que les écarts entre les deux modes 


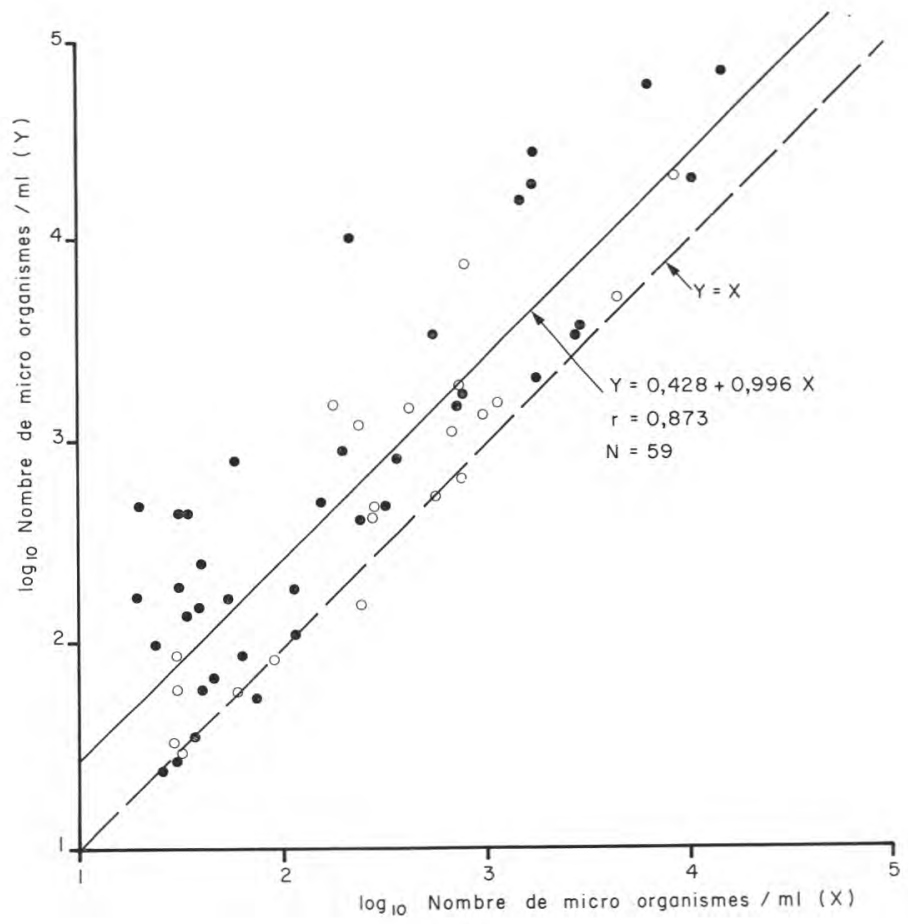

fig. 2

Bactéries coliformes (pour la légende voir fig. 1).

Coliform bacteria (as to fig. 1).

d'agitation sont intermédiaires entre ceux trouvés pour la flore thermorésistante et pour la flore psychrotrophe résistant à la pénicilline : ils sont en général inférieurs à 0,85 unité de $\log$ (à $\mathrm{P}=0,95$ ) avec une moyenne d'environ 0,30 unité de log, soit une multiplication moyenne par 2 des nombres de micro-organismes. L'écart que nous avons trouvé est d'ailleurs nettement inférieur à celui qu'avaient observé Te Whaiti et Fryer (1977) : d'après ces derniers, les nombres de micro-organismes étaient en moyenne multipliés par 6 après agitation avec la seringue. Ce désaccord entre auteurs peut être attribué à des différences entre échantillons. D'autre part, l'effet de l'agitation des échantillons sur les résultats de dénombrement de la flore totale semble légèrement varier en fonction du niveau de contamination (pente de la droite de régression légèrement supérieure à 1). Cela peut résulter de la variation de la nature de la flore du lait en fonction du niveau de contamination : en effet, les échantillons peu contaminés contiennent en général, en flore dominante, des microcoques et des streptocoques ; par contre, la proportion de bactéries à Gram négatif 


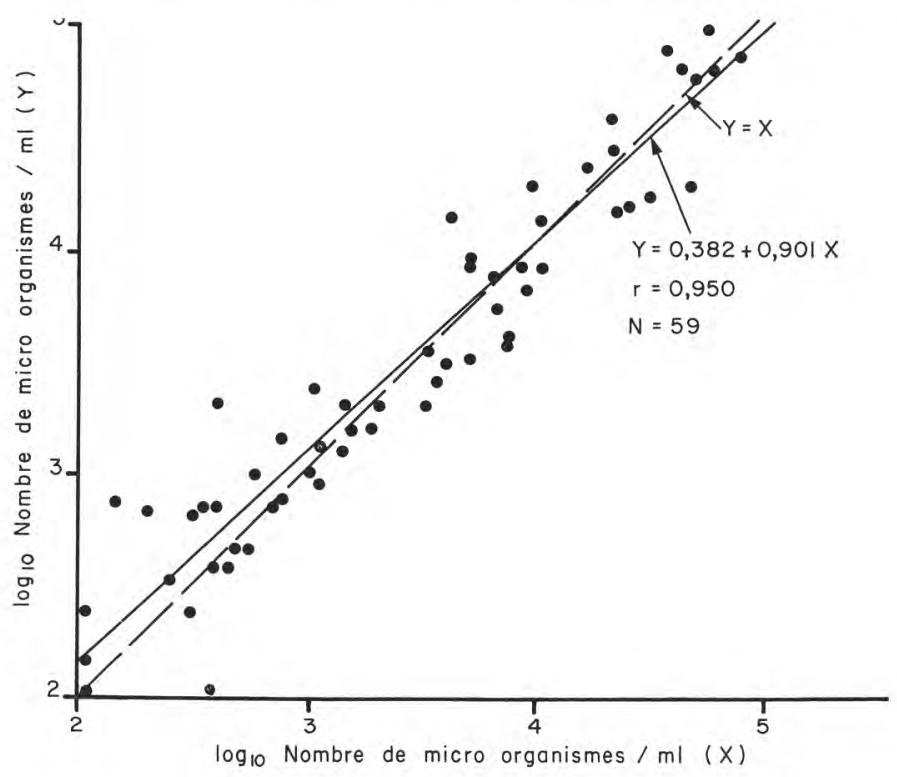

fig. 3

Flore thermorésistante totale (pour la légende voir fig. 1).

Thermoduric flora (as to fig. 1).

devient très importante dans les laits très pollués (Thomas et al., 1962 ; Twomey et Crawley, 1968) ; les Pseudomonas dominent la flore totale des laits conservés longtemps à basse température (Richard, 1981). Il en résulte que l'effet de l'agitation devrait être d'autant plus important que les échantillons sont plus contaminés.

\section{CONCLUSION}

L'influence du mode d'agitation sur les résultats de dénombrement est très importante dans le cas de la flore à Gram négatif (flore psychrotrophe résistant à la pénicilline et bactéries coliformes) ; elle est par contre imperceptible pour la flore thermorésistante. Cela est sans doute lié au fait que les amas formés par les bactéries à Gram négatif sont plus faciles à dissocier que ceux formés par les bactéries à Gram positif, lesquels dominent dans la flore thermorésistante (Thomas et al., 1950 ; Thomas et al., 1967). Il en résulte que l'écart entre les modes d'agitation, pour la flore totale, varie en fonction de la nature des bactéries qui la composent, comme l'avaient déjà observé Te Whaiti et Fryer (1977) mais pour 18 échantillons seulement. 


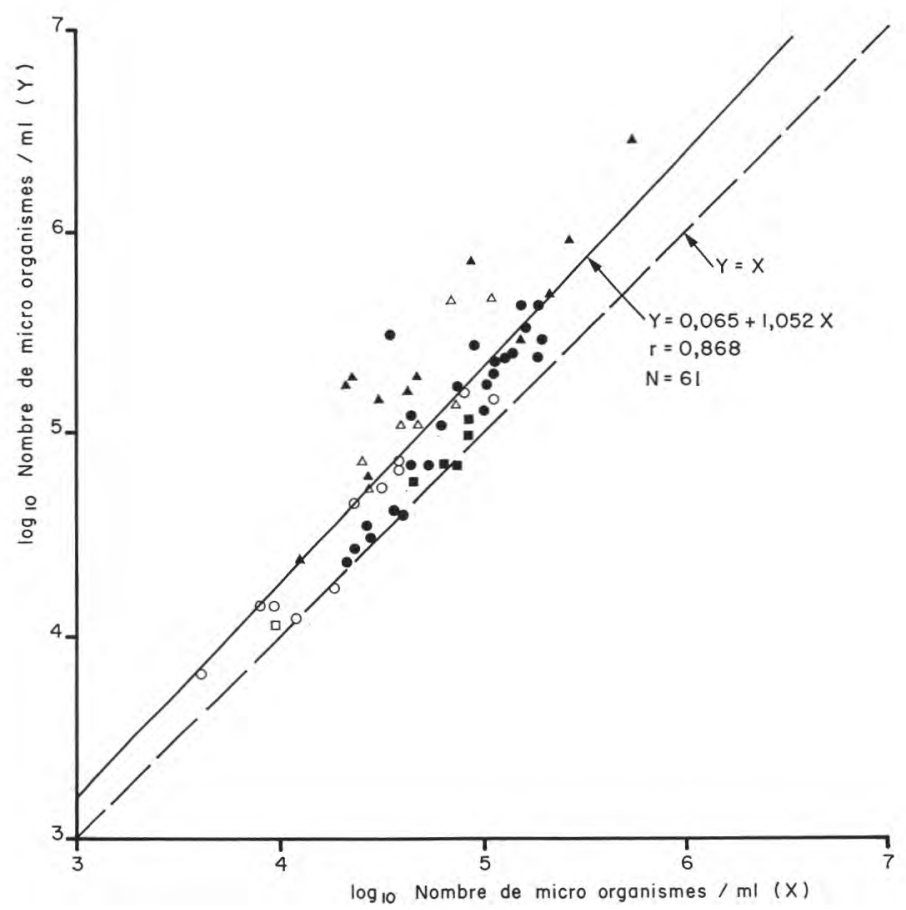

fig. 4

Flore totale - Triangles et carrés : respectivement forte proportion, dans la flore totale, de bactéries psychrotrophes résistant à la pénicilline et de bactéries thermorésistantes (pour le reste de la légende, voir fig. 1).

Total count - Triangles and squares: respectively high proportion of psychrotrophic penicillin resistant bacteria and thermoduric bacteria in total count (for further explanation see fig. 1).

Pour la flore psychrotrophe résistant à la pénicilline et pour les bactéries coliformes du lait, un mode d'agitation peu énergique aboutira souvent à une très nette sous-estimation du nombre de ces bactéries présentes dans le lait (souvent 20 et parfois près de 100 fois moins). Or, la conservabilité du lait à basse température dépend essentiellement de sa teneur initiale en Pseudomonas, lesquels constituent la majeure partie des bactéries psychrotrophes résistant à la pénicilline du lait cru (Richard, 1981). En prenant un taux moyen journalier de multiplication des Pseudomonas de 10 , à $4-5^{\circ} \mathrm{C}$ (Richard, 1982) cette sous-estimation peut donc entraîner un jugement erroné sur la qualité de conservation d'un lait. En effet, si l'on conclut sur la base d'une faible agitation qu'un lait contient 1000 Pseudomonas/ $\mathrm{ml}$ alors qu'il en renferme 20 fois plus, après $48 \mathrm{~h}$ à $4-5^{\circ} \mathrm{C}$ il atteindra 
2 millions de cellules/ml, soit un ordre de grandeur à partir duquel des défauts de qualité du lait et des produits laitiers sont observés (Patel et Blankenagel, 1972 ; Punch et al., 1965 ; Dumont et al., 1977) alors qu'on en prédira seulement 100000 . Il semble donc intéressant de préconiser, pour le dénombrement de ces micro-organismes, un mode d'agitation des échantillons plus énergique que ceux habituellement pratiqués par les laboratoires : une agitation par turbine ou un mode d'agitation plus facilement applicable comme peut-être l'utilisation d'une seringue proposée par Te Whaiti et Fryer (1977).

\section{Remerciements}

Ce travait a été réalisé en 1981-1982 dans le cadre d'une étude pilote sur le «Diagnostic des fautes d'hygiène à la production à partir de l'analyse bactériologique d'un échantillon de lait de tank".

Nous remercions la Fédération Nationale des Producteurs de Lait d'avoir financé cette étude et le Laboratoire Interprofessionnel Laitier de la Sarthe pour l'envoi des échantillons de lait.

\section{Bibliographie}

ANONYME (1972). - Standard methods for the examination of dairy products. American Public Health Association, 13th ed., Washington (U.S.A.).

Bottazi (V.), Dellaglio (F.) and Sarra (P. G.) (1972). - Creaming of milk and bacterial agglutination of microorganisms. IV. Mechanism causing fat and bacterial agglutination. Scienza e Technica Lattiero-Casearia, 23, 287-304

CAMpbell (J. E.) and GILChrist (J. E.) (1973). - Spiral plate technique for counting bacteria in milk and other foods. Dev. Ind. Microbiol., 14, 95-102.

Donnelly (C. B.), Gilchrist (J. E.), Peeler (J. T.) and Campbell (J. E.) (1976). Spiral plate count method for the examination of raw and pasteurized milk. Applied Environmental Microbiology, 32, 21-27.

Dumont (J. P.), Delespaul (G.), Miguot (B.) et AdDa (J.) (1977). - Influence des bactéries psychrotrophes sur les qualités organoleptiques de fromages à pâte molle. Le Lait, 57, 619-630.

Gilchrist (J. E.), Campbell (J. E.), Donnely (C. B.), Peeler (J. T.) and Delaney (J. M.) (1973). - Spiral plate method for bacterial determination. Applied Microbiology, 25, 244-252.

Huhtanen (C. N.), Brazis (A. R.), Arledge (W. L.), Cook (E. W.), Donnely (C. B.), GinN (R. E.), Murphy (J. N.), Randolph (H. E.), Sing (E.L.) and Thompson (D. I.) (1970). - Effect of dilution bottle mixing methods on plate counts of raw milk bacteria. Journal of Milk and Food Technology, 33, 269-273.

JARViS (B.), LACH (V. H.) and Wood (J. M.) (1977). - Evaluation of the spiral plate maker for the enumeration of microorganisms in foods. Journal of Applied Bacteriology, 43, 149-157

Patel (G. B.) and Blankenagel (G.) (1972). - Bacterial counts of raw milk and flavor of the milk after pasteurization and storage. J. Milk Food Technol., $35,203-206$.

Peeler (J. T.), Gilchrist (J. E.), Donnelly (C. B.) and Campbell (J. E.) (1977). - A collaborative study of the spiral plate method for examining milk samples. Journal of Food Protection, 40, 462-464. 
Punch (J. D.), Olson (J. C. Jr) and Thomas (E. L.) (1965). - Psychrophilic bacteria. III. Population levels associated with flavor or physical change in milk. J. Dairy Sci., 48, 1179-1183.

RICHARD (J.) (1980). - Influence de l'agitation du lait cru sur les résultats de dénombrement de sa flore totale à l'aide d'une anse calibrée. Le Lait, 60, 211-225.

RICHARD (J.) (1981). - Influence de diverses méthodes de nettoyage des machines à traire sur la «qualité de conservation » du lait cru à basse température. Le Lait, 61, 354-369.

RICHARD (J.) (1982). - Taxonomy and ecology of raw milk psychrotrophic Pseudomonas. In "Psychrotrophic microorganisms in spoilage and pathogenicity». XI ICFMH Symposium Aalborg, 1980, Academic Press Inc. London.

Richard (J.), Krebs (C.), Walter (J.) et Grappin (R.) (1981). - Comparaison de divers modes d'agitation des échantillons de lait cru en vue de la numération de sa flore totale suivant la méthode Thompson. Le Lait, 61, 250-260.

TAtini (S. R.), Dabbah (R.) and Olson (J. C. Jr) (1967). - Comparison of plate loop and agar plate methods for bacteriological examination of manufacturing grade raw milk. Journal of milk and Food Technology, 30, 112-115.

Te Whatti (I. E.) and Fryer (T.F.) (1977). - The enumeration of bacteria in refrigerated milk. New Zealand Journal of Dairy Science and Technology, 12, 51-57.

Thomas (S. B.), Egdell (J. W.), Clegg (L. F. L.) and Cuthbert (W. A.) (1950), Thermoduric organisms in milk. Part I. A review of the literature. Proceedings of the Society for Applied Bacteriology, 13, 27-64.

Thomas (S. B.), Hobson (P. M.), Bird (E. R.), King (K. P.), Druce (R, G.) and Cox (D. R.) (1962). - The microflora of raw milk as determined by plating on Yeastrel Milk Agar incubated at $30^{\circ} \mathrm{C}$. Journal of Applied Bacteriology, 25, 107-115.

Thomas (S. B.), Druce (R. G.), Peters (G. J.) and Griffiths (D. G.) (1967). Incidence and significance of thermoduric bacteria in farm milk supplies. A reappraisal and review. Journal of Applied Bacteriology, 30, 265-298.

Twomey (A.) and CRAWley (W. E.) (1968). - The microflora of raw milk. New Zealand Journal of Dairy Technology, 3, 44-48.

Wanser (B. E.) and Hartman (P. A.) (1962). - Agitation of milk samples for colony counts of raw milk. Journal of Milk and Food Technology, 25, 5-6. 